\title{
A Re-assessment of 4CMenB Vaccine Effectiveness Against Serogroup B Invasive Meningococcal Disease in England based on an Incidence Model
}

Lorenzo Argante ( $\sim$ lorenzo.x.argante@gsk.com )

GSK Vaccines S.r.l.: GSK Vaccines SRL https://orcid.org/0000-0001-6853-068X

Victoria Abbing-Karahagopian

GSK Vaccines S.r.l.: GSK Vaccines SRL

Kumaran Vadivelu

GSK Vaccines SRL

Rino Rappuoli

GSK Vaccines SRL

Duccio Medini

GSK Vaccines SRL

Research article

Keywords: invasive meningococcal disease, IMD, MenB, 4CMenB, Bexsero, effectiveness, VE, incidence model, screening method, epidemiology

Posted Date: May 6th, 2021

DOl: https://doi.org/10.21203/rs.3.rs-493163/v1

License: (c) (1) This work is licensed under a Creative Commons Attribution 4.0 International License.

Read Full License

Version of Record: A version of this preprint was published at BMC Infectious Diseases on December 1st, 2021. See the published version at https://doi.org/10.1186/s12879-021-06906-x. 
A re-assessment of $4 C M e n B$ vaccine effectiveness against serogroup $B$ invasive meningococcal disease in England based on an incidence model

Lorenzo Argante $^{1 *}$, Ph.D., Victoria Abbing-Karahagopian², Ph.D., Kumaran Vadivelu¹, M.B., B.S., Rino Rappuoli ${ }^{1}$, Ph.D., and Duccio Medini ${ }^{1}$, Ph.D.

${ }^{1}$ GSK, Siena, Italy

${ }^{2} \mathrm{GSK}$, Amsterdam, the Netherlands

*Corresponding author: lorenzo.x.argante@gsk.com

Email addresses:

lorenzo.x.argante@gsk.com

victoria.x.abbing-karahagopian@gsk.com

kumaran.k.vadivelu-pechai@gsk.com

rino.r.rappuoli@gsk.com

duccio.medini@gmail.com 


\section{ABSTRACT}

Background: The four-component serogroup B meningococcal 4CMenB vaccine (Bexsero, GSK) has been routinely given to all infants in the United Kingdom at 2, 4 and 12 months of age since September 2015. After three years, Public Health England (PHE) reported a 75\% [95\% confidence interval: $64 \% ; 81 \%$ ] reduction in the incidence of serogroup B invasive meningococcal disease (IMD) in age groups eligible to be fully vaccinated. In contrast, vaccine effectiveness (VE) evaluated in the same immunization program applying the screening method was not statistically significant. We reanalyzed the data using an incidence model and obtained more precise VE estimates.

Methods: Aggregate data - stratified by age, year and doses received - were provided by PHE: serogroup B IMD case counts for the entire population of England (years 2011-2018) and 4CMenB vaccine uptake in infants. We combined uptake with national population estimates to obtain counts of vaccinated and unvaccinated person-time by age and time. We re-estimated VE comparing incidence rates in vaccinated and non-vaccinated using a Bayesian Poisson model for case counts with person-time data as an offset. The model was adjusted for age, time and number of doses received. Results: The incidence model showed that cases decreased until 2013-2014, followed by an increasing trend that continued in the non-vaccinated population during the immunization program. VE in fully vaccinated (three doses) was $80.1 \%$ [95\% Bayesian credible interval (BCI): $70.3 \%$; 86.7\%]. After a single dose, VE was 33.5\% [12.4\%; 49.7\%] $95 \% \mathrm{BCl}$, and, after two doses, $78.7 \%$ $[71.5 \% ; 84.5 \%]_{95 \% \mathrm{BCl}}$. We estimated that vaccination has averted 312 cases $[252 ; 368]_{95 \% \mathrm{BCl}}$ between 2015 and 2018. VE was in line with the previously reported incidence reduction.

Conclusions: Our estimates of VE had higher precision than previous estimates based on the screening method, that were not statistically significant. When disease incidence is low and vaccine uptake is high, the screening method applied to cases exclusively from the population eligible to vaccination may not be enough precise and produce misleading point-estimates. Precise and accurate VE estimates are fundamental for informing public health decision making. VE assessment can be enhanced using models that leverage data on subjects not eligible for vaccination.

Keywords: invasive meningococcal disease, IMD, MenB, 4CMenB, Bexsero, effectiveness, VE, incidence model, screening method, epidemiology. 


\section{BACKGROUND}

Neisseria meningitidis is a strictly human bacterium that can cause invasive meningococcal disease (IMD). The most frequent clinical manifestations of IMD are meningitis and sepsis, serious and rapidly fulminant conditions associated with considerable mortality and sequalae worldwide. ${ }^{1-3}$ There are six major serogroups $(A, B, C, W, X$, and $Y)$ responsible for most IMD cases and the disease incidence is generally highest in infants, showing geographically asynchronous secular trends leading to substantial temporal variability in the number of cases. ${ }^{2,4}$ Serogroup B is currently a major cause of IMD in the Americas, Australia and Europe. ${ }^{5}$

The four-component protein vaccine 4CMenB (Bexsero, GSK) is the only broadly protective vaccine against serogroup B IMD licensed by the European Medicines Agency for use in infants. ${ }^{6}$ Prelicensure efficacy trials for meningococcal vaccines are not feasible because the incidence of IMD is low. Hence, $4 \mathrm{CMenB}$ licensure was based on studies testing its safety and immunogenicity, which is measured through a serum bactericidal assay with human complement (hSBA) that correlates with protection. ${ }^{7}$ Accordingly, real-world studies of vaccine effectiveness and impact are deemed very important.

The first of such real-world study looking into 4CMenB vaccine effect was conducted by Public Health England (PHE). In September 2015, the United Kingdom became the first country to include 4CMenB in its national immunization program offering a reduced schedule of three doses of $4 \mathrm{CMenB}$ to all infants: two primary doses at 2 and 4 months of age and a booster at age 12 months. ${ }^{8}$ After three years of $4 \mathrm{CMenB}$ vaccination, PHE reported a very high vaccine uptake $(92.5 \%$ for the primary twodoses immunization and $87.9 \%$ for the third dose) and a statistically significant $75 \%$ reduction of the incidence of serogroup B IMD (95\% confidence interval [Cl]: [64\%; 81\%]). This vaccine impact was estimated on age groups fully eligible for vaccination (that included also non-vaccinated and partially vaccinated subjects) through a Poisson model. ${ }^{9}$

In contrast, the same study reported vaccine effectiveness (VE) estimates which were lower than the vaccine impact and not significantly different from zero (e.g., in fully vaccinated with three doses, $\left.59.1 \%[-31.1 \% ; 87.2 \%]_{95 \% \mathrm{Cl}}\right) .^{9} \mathrm{VE}$ was estimated using the screening method, an approach that 
expresses the effectiveness as a function of the proportion of cases that has been vaccinated and the vaccine uptake in the population. ${ }^{10-12}$

VE lower than vaccine impact appears inconsistent because the latter was measured on a population that included non-vaccinated. Indeed, when indirect effects of a vaccine are absent or negligible, especially at the beginning of an immunization program, vaccine impact $(\mathrm{VI})$ should theoretically equal its effectiveness multiplied by the proportion of vaccinated persons (i.e., the vaccine uptake $x$ ): $V I=V E * x$. This simple mathematical relation has been previosly used to broadly estimate VE from impact and uptake,,$^{13}$ and we provide its formal derivation in supplementary section S1. Since $x$ cannot be higher than one (i.e., 100\% uptake), it follows that VE should be at least as high as VI. Therefore, undefined effectiveness (not significantly greater than zero) appears inconsistent with the statistically significant $75 \%$ impact reported by $\mathrm{PHE} .{ }^{9}$

The inconsistency between the reported impact and VE may be due to the use of screening method for VE estimation. This warrants a rigorous re-estimation of $4 \mathrm{CMenB}$ effectiveness with greater precision. We analyzed real-world data on serogroup B IMD and 4CMenB uptake between September 2011 and August 2018 provided to us by PHE, and re-assessed the effectiveness of one to three doses of $4 \mathrm{CMenB}$ using an incidence model.

\section{METHODS}

\section{Disease cases and vaccine uptake}

All the data on IMD disease and vaccine uptake were provided to us by Public Health England (PHE). Detailed information on the implementation of the immunization program, on PHE's routine surveillance of patients with IMD in England and on uptake data collection are available in the original publication by Ladhani and collaborators. $^{9}$

The data on IMD that we received (supplementary table S1) were case counts of laboratoryconfirmed serogroup B IMD cases from the entire population of, aggregated by age (eleven age groups, 0-1 months the first, older than 44 years the last), by year of disease onset, and by number of $4 \mathrm{CMenB}$ doses received at least fourteen days before disease onset (fourteen days is the time to 
mount an immune response). The time period spanned seven years, from September 2011 through August 2018. Time units were years (from September through the following August). 4CMenB vaccination started in September 2015, therefore the data covered four pre-vaccination years and three years during the immunization program. The number of doses received ranged from zero, i.e., unvaccinated, to three, i.e., fully vaccinated. Before September 2015 all the cases were unvaccinated.

The data on vaccine uptake provided by PHE were: i) proportions of infants eligible to the immunization program that received one to three doses of $4 \mathrm{CMenB}$ at 6,12 and 18 months of age, by month of time; ii) the same proportions by day of age and day of time for nearly 60,000 individuals $(30,000$ for the third dose) from different geographical areas across England. We used both the data sources, that were combined together following the same approach used in the original study by Ladhani and collaborators, ${ }^{9}$ as described in supplementary section S3.

In addition to the above described data, we used population estimates for England (years 2011-2018) from the Office for National Statisitcs of the United Kingdom (publicly available at https://www.ons.gov.uk). We grouped the population estimates in the same age-and-time structure as the IMD case counts. Then, we combined population estimates with the uptake proportions to derive the number of person-years for each age group defined by PHE, for each year (from September through August) and for each number of doses received at least fourteen daysearlier. Details are provided in supplementary section S3, person-years are plotted in supplementary figures S1 and S2.

\section{Model-based vaccine effectiveness re-assessment}

The effectiveness of $4 \mathrm{CMenB}$ was re-evaluated comparing serogroup $\mathrm{B}$ IMD incidence rates $I R$ in vaccinated with $I R$ in non-vaccinated: ${ }^{12}$

$$
V E=\frac{I R_{\text {unvaccinated }}-I R_{\text {vaccinated }}}{I R_{\text {unvaccinated }}}=1-I R R,
$$

where $I R R$ is an incidence rate ratio. 
Since the data were counts, we used a Poisson model. ${ }^{14}$ We employed a Bayesian approach. ${ }^{15,16}$ Bayesian methods have been already used to estimate VE. ${ }^{17}$ Specifically, we modelled counts of serogroup B IMD cases $y_{a, t, d}$ with a hierarchical Poisson regression model, ${ }^{15}$ adjusting for age $a$, year $t$ and number of vaccine doses $d$, using person-time $N_{a, t, d}$ as an offset:

$$
\begin{aligned}
y_{a, t, d} & \sim \operatorname{Poisson}\left(N_{a, t, d} e^{\rho_{a}+\beta_{t}+\theta_{d}}\right), \\
\beta_{t} & \sim \operatorname{Normal}\left(0 ; \sigma_{\beta}^{2}\right),
\end{aligned}
$$

where $e^{\rho_{a}}$ is the average incidence rate in the unvaccinated population in age group $a$. The parameter $\beta_{t}$ adjusts for time, while $\theta_{d}$ controls for the number of doses $d$ received at least two weeks before. $\beta_{t}$ was constrained to zero sum: their values must be considered relatively to the average $\bar{\beta}$ across the full study period, i.e. $\bar{\beta}$ is the baseline for $\beta_{t}$.

With this parametrization, $e^{\rho_{a}+\beta_{t}}$ is the incidence rate in unvaccinated subjects, corrected for age and year. We fixed $\theta_{0}=0$ as a baseline for $\theta$, so that $e^{\theta_{d}}$ is the age-and-time-adjusted incidence rate ratio $I R R_{d}$ in vaccinated subjects that received $d>0$ doses relative to unvaccinated subjects. Therefore, consistently with the VE definition, the effectiveness of $d$ doses was:

$$
V E_{d}=1-I R R_{d}=1-e^{\theta_{d}} .
$$

Since we fixed $\theta_{0}=0$, it follows that $V E_{0}=0$, meaning that the vaccine was assumed to have no effect on unvaccinated groups, i.e. when $d=0$.

We assigned non-informative prior distributions to all the model's parameters and numerically estimated the respective posterior distributions, given prior, data and model. We chose noninformative priors so that posterior values are affected only by data and model through the assumed Poisson likelihood. Bayesian estimation was numerically executed using Markov chain Monte Carlo. Specifically, we used a Hamiltonian Monte Carlo algorithm: the No-U-Turn sampler of Python's PyMC3 package (further details in supplementary section S4). ${ }^{18}$ Posterior distributions of the parameters were summarized through their point estimates (posterior means) and the limits of their 95\% Bayesian credible interval $(95 \% \mathrm{BCl}$, calculated as the $95 \%$ highest posterior density). VEs were derived from $\theta$ through equation 3 . 


\section{Expected cases, counterfactual and averted cases}

To estimate of number of averted cases we used the model to generate a counterfactual (i.e. the expected case counts if no immunization program was implemented), and compared it with the expected case counts from model's best fit on the observed data. In practice, posterior distributions of expected cases were numerically generated randomly sampling from the fitted model $(100,000$ iterations). Counterfactuals were sampled in the same manner, except that all the effectiveness parameters ( $\theta$ in the model) were fixed to zero, to simulate the absence of vaccination. Probability distributions of the averted cases imputable to the vaccine were calculated subtracting counterfactuals from best-fitted expected cases. All the distributions were summarized and reported as mean and $95 \% \mathrm{BCl}$. Further details are reported in supplementary section S5.

\section{Expected VE using the mathematical relation between impact, uptake and VE}

When indirect effects are absent or negligible, the impact of a vaccine equals VE multiplied by the vaccine uptake. ${ }^{13}$ For a vaccine three doses, the formula becomes (full derivation in supplementary section S1):

$$
V I=x_{1} V E_{1}+x_{2} V E_{2}+x_{3} V E_{3},
$$

where VI is the vaccine impact. $x_{1}, x_{2}$ and $x_{3}$ are the proportions of subject vaccinated with one to three doses.

\section{RESULTS}

\section{Estimates of incidence rates and vaccine effectiveness}

The re-analysis of data on IMD cases 4 CMenB uptake using an incidence model allowed us to obtain point estimates and Bayesian credible intervals for four relevant measures: (i) average IMD incidence rates per age-group in non-vaccinated subjects in the period from September 2011 through August 2018, (ii) the variation of incidence rates over time in non-vaccinated relatively to their baseline, (iii) incidence rate ratios in vaccinated with respect to non-vaccinated, and (iv) VE of 4 CMenB. Supplementary table S2 reports the list of all the fitted parameters.

Figure $1 A$ shows that serogroup $B$ IMD incidence rates per age-group in the non-vaccinated population (2011-2018 average) were in line with previous observations: ${ }^{19}$ the highest incidence was 
in infants (especially at four to eleven months of age); a relative maximum was observed in the 15-24 years age group; an increase was found in the last age group that included the elderly.

Incidence rates in the non-vaccinated population varied significantly across years, relatively to the 2011-2018 average, exhibiting a decrease followed by an increasing trend (Figure 1B). Incidence was highest during the first year 2011-2012 (1.16 times the average), then it declined and reached its minimum in 2013-2014 (0.84 times the average). Thereafter, before and during the immunization program, the relative incidence in non-vaccinated continuously increased, up to 1.07 times the average in $2017-2018$, which was 1.25 times significantly higher $\left([1.09 ; 1.43]_{95 \% \mathrm{BCl}}, \mathrm{P}<0.001\right)$ than the incidence observed during the last pre-vaccination year, i.e., 2014-2015.

Figure $1 \mathrm{C}$ shows the incidence rate ratios obtained from the best fit of the model to data. Our estimates of the effectiveness of $4 C M e n B$ (Figure $2 A$ ) show that VE was significantly higher than zero $\left(P_{\mathrm{VE} \leq 0}=0.0014\right)$ already after a single dose: $33.5 \%,[12.4 \% ; 49.7 \%]_{95 \% \mathrm{BCI}}$. After two doses vaccine effectiveness was $78.7 \%[71.5 \% ; 84.5 \%]_{95 \% \mathrm{BCI}}(\mathrm{P}<0.001)$. VE after the full three-dose schedule was $80.1 \%,[70.3 \% ; 86.7 \%]_{95 \% \mathrm{BCl}}(\mathrm{P}<0.001)$. Subjects that received only the first dose were nearly three times more at risk of disease than those who received two doses (incidence rate ratio: $\left.3.13,[2.11 ; 4.62]_{95 \%} \mathrm{BCl}\right)$. Figure $2 \mathrm{~B}$ shows for comparison $\mathrm{VE}$ values estimated using the screening method. ${ }^{9}$

\section{The fitted model accurately reproduced observed IMD case counts}

Figure 3 shows the number of serogroup B IMD cases per year and age group, reported in England between September 2011 and August 2018, along with the best fit of the incidence model to data (blue curves). Supplementary figure S3 reports the same data and expected cases from model's fit stratified also by number of $4 \mathrm{CMenB}$ doses received. The model accurately reproduced disease cases in each year, for every age group and number of doses received (supplementary figure S4 and section $\mathrm{S} 6, \mathrm{R}^{2}$ predicted $v$ s. observed $>0.96$ ). Supplementary table $\mathrm{S} 3$ shows that the incidence model achieved better accuracy than an alternative implementation not adjusting for time. 


\section{Countefactual estimates and averted cases}

Counterfactuals - i.e., expected numbers of serogroup B IMD cases that would have occurred in England if $4 \mathrm{CMenB}$ was not included in the national immunization program - are shown in figure 3 and supplementary figure S3 as red dashed curves. We calculated an excess of $312[252 ; 368]_{95 \% \mathrm{BCl}}$ cases prevented by the campaign during its first three years. Specifically, 46 [30;62] during its first year, $113[84 ; 140]$ during the second and $153[117 ; 186]$ during the third year.

\section{Our estimates of VE are in line with the impact reported in the original study}

The uptake of one, two and three doses in the fully eligible age groups on which PHE measured the vaccine impact were, respectively, $x_{1}=3.6 \%, x_{2}=4.6 \%, x_{3}=87.9 \% .{ }^{9}$ Substituting these values and our estimates of VE in the mathematical expression relating impact, uptake and VE for vaccinations with three doses (equation 4), we find $\mathrm{VI}=75.2 \%[65.5 \% ; 81.9 \%]$ 95\%BCI, in agreement with $\mathrm{VI}$ estimated by PHE.

\section{DISCUSSION}

In this re-assessment we showed that that the effectiveness of $4 \mathrm{CMenB}$ vaccine has been $80.1 \%$ $[70.3 \% ; 86.7 \%]_{95 \% \mathrm{BCl}}$ in fully vaccinated infants during its first three years of implementation in a national immunization program in England. We demonstrated that our VE estimates are in line with the previously reported vaccine impact: a $75 \%[64 \% ; 81 \%]_{95 \% \mathrm{Cl}}$ reduction of serogroup B IMD incidence in age groups fully eligible for vaccination. ${ }^{9}$

The same study ${ }^{9}$ reported VE estimates inconsistently lower than the impact even in fully vaccinated, and not statistically significant. They were calculated using the screening method, a simple and rapid approach where VE is estimated from the proportion of cases that are vaccinated and the proportion of population vaccinated. ${ }^{10-12}$ As stated by the authors who proposed it in 1985 , this method is a rapid mean to obtain preliminary estimates, should not be relied upon for precise VE estimates, and more accurate techniques are needed if the effectiveness is lower than expected levels. ${ }^{10}$

The lack of precision of the estimates based on the screening method compared to our reassessment, may be due to the fact that the screening method relies exclusively on cases emerging 
from the population eligible for vaccination. When the disease incidence is low and the vaccine uptake is high - as is the case in this study - the number of unvaccinated cases in the eligible population may be very low or even null (depending on the observational time), thus inadequate to produce sufficiently precise VE estimates. ${ }^{20}$ Instead, our incidence model used non-vaccinated cases from the eligible as well as from the non-eligible population to achieve higher precision.

Though all our point estimates for VE fall inside the respective $95 \%$ confidence intervals obtained via screening method, point estimates produced with the screening approach are consistently lower than our results. The reason for this difference may be another consequence of the small number of cases that already caused the lack of precision. Indeed, the screening method was applied matching the proportion of vaccinated to the observed cases, according to number of doses received, age and year at disease onset. As the number of disease cases was extremely small compared to the population eligible to the campaign, the matched vaccine uptake may not have been representative of the vaccine uptake in the population, especially with disease incidence and uptake per dose greately varying with age, as observed in this immunization program. Therefore, the low number of cases and a possible unfortunate uptake matching may have biased VE estimates towards lower values. In theory, it could have also led to the opposite effect, i.e. an overestimated VE, depending on age and time at disease for the limited number of cases to which the uptake was matched. The screening method would have likely required more years of surveillance to have a sufficient number of cases for an accurate evaluation of the proportion of vaccinated population. Instead of using only uptake data relative to cases, we used more robust person-time data of the whole vaccinated and unvaccinated population (for the largest majority composed by non-cases) and could estimate VE more accurately and in line with the previously reported incidence reduction. ${ }^{9}$

Most relevant from a public health perspective is the overall number of averted cases, that we estimated to be $312[252-369]_{95 \% \mathrm{BCl}}$, which is in good agreement with the 277 [236 - 323 $]_{95 \% \mathrm{Cl}}$ averted cases estimated by $\mathrm{PHE}^{9}{ }^{9}$ Disease incidence decreased during the first two pre-vaccination years here considered, then slowly inverted its trend, started to rise, and continuously increased - in non-vaccinated - after the inclusion of $4 \mathrm{CMenB}$ in England's infant national immunization program in 
September 2015 (Figure 1, panel B). Thus the decision to include 4CMenB in the national immunization program was timely.

Althought our VE estimates were more precise than screening method's and in agreement with the incidence reduction, also our analysis has some limitations. The data on vaccination used in this reassessment were originally collected to apply the screening method. Therefore, information on vaccination status at individual level was collected for cases only. By design, vaccination status of non-cases, i.e. controls, was derived from uptake statistics that came from a source external to the study. ${ }^{11}$ Also, data on disease that we received were case counts, already aggregated by age, year and doses. Even if we believe that the stratification was optimal for this kind of analysis, we could not test the sensitivity of the result when varying the stratification. Another possible limitation is that we could not test covariates other than age, time and doses received, to find and adjust for potential additional confounders. An intrinsic weakness of our model-based approach is that it assumes incidence rates are constant within each group. Also, not having modelled transmission to predict dynamically secular incidence trends, we assumed that yearly incidence variation would affect each age group proportionally.

Precise and accurate estimates of $4 \mathrm{CMenB}$ effectiveness are essential to appropriately inform costeffectiveness analyses that support public health decision making. ${ }^{21,22}$ Provided the availability of strain typing data ${ }^{23,24}$ on the same disease cases used for this re-assessment, the higher precision of our approach will allow to further stratify data and to assess 4 CMenB effectiveness against different strain types. Such estimates, combined with strain typing data from other countries, ${ }^{25}$ would allow even more robust predictions of $4 \mathrm{CMenB}$ effects in different geographies, improving costeffectiveness evaluations. In future, our approach may be adopted in similar settings by using surveillance data at population level during vaccination programs, if more traditional methods are underpowered.

Our results are also aligned with $4 \mathrm{CMenB}$ effectiveness in infants and children reported by two recent observational studies in Italy (two regions, Tuscany and Veneto) and Portugal. ${ }^{26,27}$ Still unclear is 4CMenB's potential impact on asymptomatic meningococcal carriage. A recent study in Australia failed to detect any effect on carriage, that could lead to indirect effects of vaccination, particularly in 
adolescents. ${ }^{28,29}$ The approach employed here, if expanded with a dynamic model of meningococcal asymptomatic transmission and invasive disease ${ }^{20,22,30}$ as done previously for evaluating a serogroup C immunization program, ${ }^{20}$ could detect indirect effects using IMD surveillance data, without or before running further meningococcal carriage studies.

\section{CONCLUSIONS}

Our analysis quantified the real-world effectiveness of $4 \mathrm{CMenB}$ during a national infant immunization program and confirmed evidence of protection from serogroup B invasive disease. The screening method may be inadequate in settings characterized by high vaccine uptake and low disease incidence, whereas carefully calibrated incidence models that more extensively and efficiently use the same kind of surveillance data may be more appropriate to assess effectiveness of meningococcal vaccines.

\section{LIST OF ABBREVIATIONS}

VE: vaccine effectiveness

VI: vaccine impact

IMD: invasive meningococcal disease

hSBA: serum bactericidal assay with human complement

PHE: Public Health England

$\mathrm{Cl}$ : confidence interval

$\mathrm{BCl}$ : Bayesian credible interval 


\section{FIGURES}

\section{Figure 1. Incidence rates}

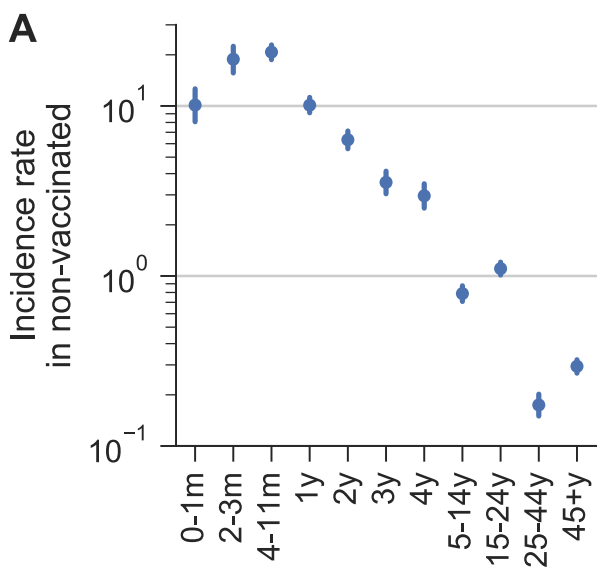

Age group

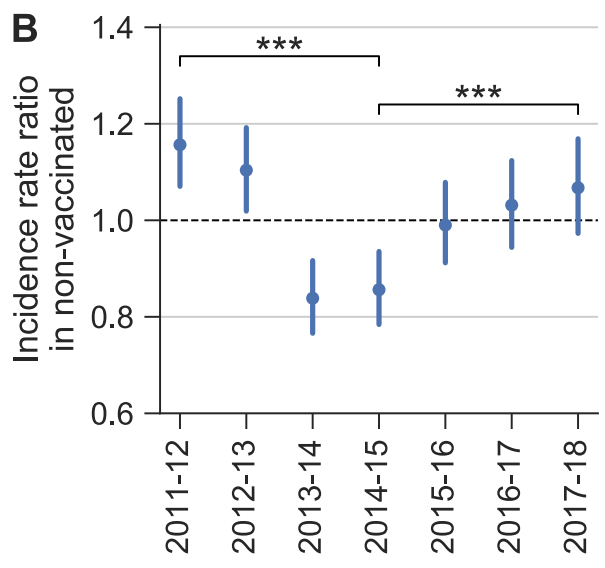

Year (September-August)

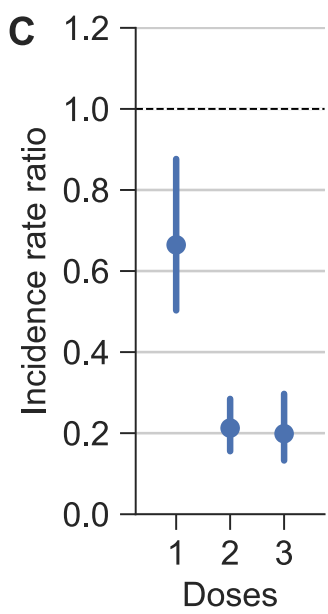

In panel A, shown are incidence rates by age in the non-vaccinated population, averaged over the period from September 2011 to August 2018 (cases per 100,000 person-years, $\mathrm{e}^{\rho_{\mathrm{a}}}$ in the model; $m=$ months, $y=y e a r s)$. In panel $B$, shown are yearly incidence rates ratios ( $\mathrm{e}^{\beta_{\mathrm{t}}}$ in the model) in the non-vaccinated population (that is the whole population for the four years before immunization, i.e., from 2011 through 2015), calculated relatively to the 2011-18 average (used as a baseline, fixed to 1 , shown as a dashed horizontal line). In panel C, shown are incidence rate ratios in vaccinated, relatively to non-vaccinated, by number of doses received ( $e^{\theta_{d}}$ in the model). Bars report $95 \%$ credible intervals around point estimates. $P$ values: ${ }^{* * *} P<0.001$. 
Figure 2. Re-assessed 4CMenB effectiveness compared with previous estimates
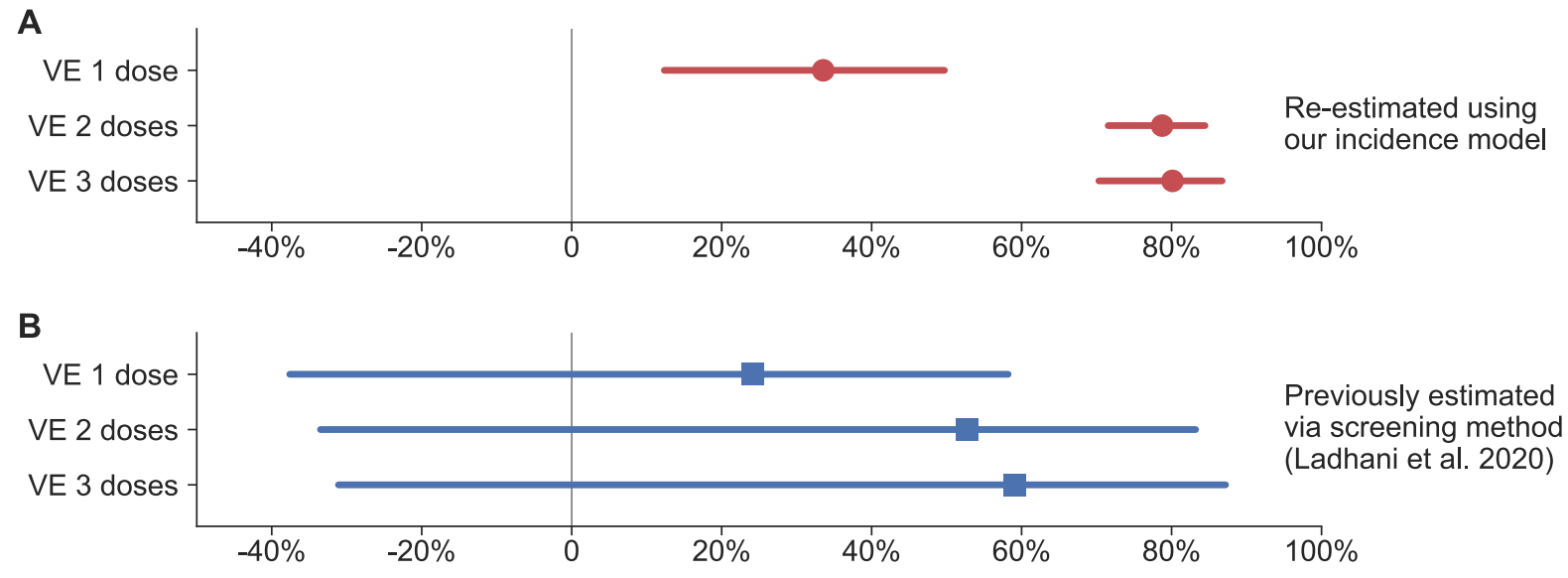

Panel $A$, in red: vaccine effectiveness (VE) of $4 C M e n B$ after one to three doses, re-estimated from reported data by Public Health England (PHE) using our Poisson regression model (Eq. 1). Panel B, in blue: VE estimated by PHE using the screening method. ${ }^{9}$ Bayesian point estimates and $95 \%$ credible intervals are marked with red circles and bars. Frequentist point estimates and 95\% confidence intervals are marked with blue squares and bars. 
Figure 3. Observed, best-fitted and counterfactual IMD case counts

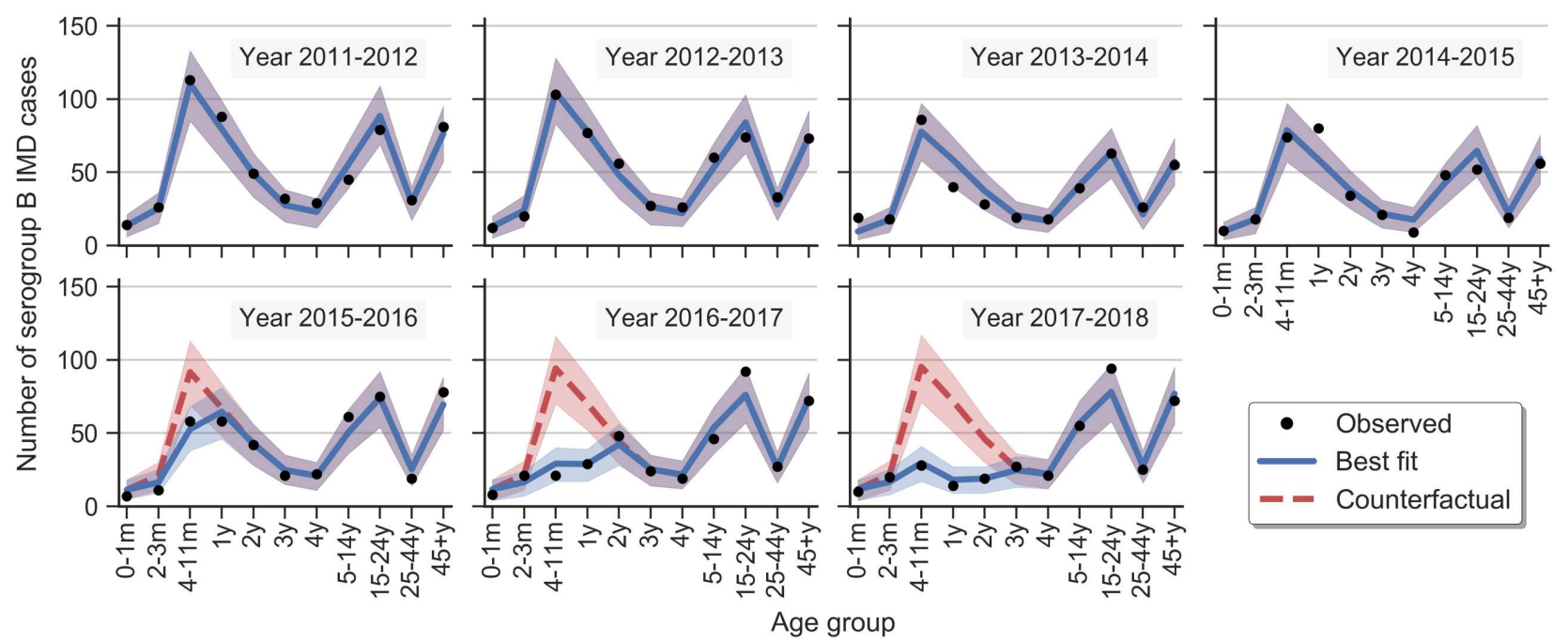

Each plot reports as black points the yearly number of observed serogroup B invasive meningococcal disease (IMD) cases by age group (the first and second row report cases before and during immunization, respectively). Incidence model's best fitted case counts are shown as blue solid lines. Red dashed lines report counterfactual IMD cases, expected in absence of vaccination, generated through the model when setting VE to zero while leaving untouched the other fitted parameters. The blue and red semi-transparent regions are the $95 \%$ credible intervals of the corresponding expected case counts. m: age in months; y: age in years. 


\section{DECLARATIONS}

\section{Ethics approval and consent to participate}

This study used epidemiological data aggregated at the country level and therefore did not require an ethics approval.

\section{Consent for publication}

Not applicable.

\section{Availability of data and materials}

The case counts of serogroub B IMD in England as used in this analysis (stratified by age, year and doses received) are provided as supplementary information (table S1).

\section{Competing interests}

Lorenzo Argante, Victoria Abbing-Karahagopian, Kumaran Vadivelu and Rino Rappuoli are employed by the GSK group of companies and declare financial and non-financial conflict of interest. Duccio Medini was employed by the GSK group of companies at the time of the study and declares financial and non-financial conflict of interest. Kumaran Vadivelu, Rino Rappuoli and Duccio Medini also hold shares in the GSK group of companies.

\section{Funding}

GlaxoSmithKline Biologicals SA was the funding source and was involved in all stages of this reassessment study. GlaxoSmithKline Biologicals SA also funded all costs associated with the development and the publishing of the present manuscript.

\section{Authors' contributions}

LA analyzed the data. All authors participated in the design of the analysis, were involved in the interpretation of the results, and the development of this manuscript. All authors had full access to the data and gave final approval before submission.

\section{Acknowledgements}

The authors would like to thank PHE Meningococcal Reference Unit \& Immunisation and Countermeasures Team for data sharing and Nick Andrews for his feedback. They also want to thanks Modis, c/o GSK, for editorial assistance and manuscript coordination (provided by Adrian 
Kremer), and medical writing support (provided by Joanne Wolter, independent medical writer c/o GSK).

\section{Trademarks}

Bexsero is a trademark owned by or licensed to the GSK group of companies.

\section{REFERENCES}

1. Stephens DS, Greenwood B, Brandtzaeg P. Epidemic meningitis, meningococcaemia, and Neisseria meningitidis. Lancet. 2007;369(9580):2196-2210. doi:10.1016/S01406736(07)61016-2

2. Acevedo R, Bai X, Borrow R, et al. The Global Meningococcal Initiative meeting on prevention of meningococcal disease worldwide: Epidemiology, surveillance, hypervirulent strains, antibiotic resistance and high-risk populations. Expert Rev Vaccines. 2019;18(1):15-30. doi:10.1080/14760584.2019.1557520

3. Black S, Pizza M, Nissum M, Rappuoli R. Toward a Meningitis-Free World. Sci Trans/ Med. 2012;4(123):123ps5-123ps5. doi:10.1126/scitransImed.3003859

4. Khatami A, Pollard AJ. The epidemiology of meningococcal disease and the impact of vaccines. Expert Rev Vaccines. 2010;9(3):285-298. doi:10.1586/erv.10.3

5. Sridhar S, Greenwood B, Head C, et al. Global incidence of serogroup B invasive meningococcal disease: A systematic review. Lancet Infect Dis. 2015;15(11):1334-1346. doi:10.1016/S1473-3099(15)00217-0

6. European Medicines Agency. Bexsero.

https://www.ema.europa.eu/en/medicines/human/EPAR/bexsero. Accessed August 3, 2020.

7. Goldschneider I, Gotschlich EC, Artenstein MS. Human immunity to the meningococcus. I. The role of humoral antibodies. J Exp Med. 1969;129(6):1307-1326. http://www.ncbi.nlm.nih.gov/pubmed/4977280.

8. Parikh SR, Andrews NJ, Beebeejaun K, et al. Effectiveness and impact of a reduced infant 
schedule of $4 \mathrm{CMenB}$ vaccine against group B meningococcal disease in England: a national observational cohort study. Lancet. 2016;388(10061):2775-2782. doi:10.1016/S01406736(16)31921-3

9. Ladhani SN, Andrews N, Parikh SR, et al. Vaccination of infants with meningococcal group B vaccine (4CMenB) in England. N Engl J Med. 2020;382(4):309-317.

doi:10.1056/NEJMoa1901229

10. Orenstein W a., Bernier RH, Dondero TJ, et al. Field evaluation of vaccine efficacy. Bull World Health Organ. 1985;63(6):1055-1068. http://www.ncbi.nlm.nih.gov/pubmed/3879673.

11. Farrington CP. Estimation of vaccine effectiveness using the screening method. Int $J$ Epidemiol. 1993;22(4):742-746. doi:10.1093/ije/22.4.742

12. Halloran ME, Longini Jr. IM, Struchiner CJ. Design and Analysis of Vaccine Studies: Introduction. Springer; 2009. http://link.springer.com/10.1007/978-0-387-68636-3.

13. Amirthalingam G, Andrews N, Keel P, et al. Evaluation of the effect of the herpes zoster vaccination programme 3 years after its introduction in England: a population-based study. Lancet Public Heal. 2018;3(2):e82-e90. doi:10.1016/S2468-2667(17)30234-7

14. Rothman, Kenneth J; Greenland, Sander; Lash TL. Modern Epidemiology. third.; 2008.

15. Gelman A, Carlin JB, Stern HS, Dunson DB, Vehtari A, Rubin DB. Bayesian Data Analysis, Third Edition. Taylor \& Francis; 2013. https://books.google.it/books?id=ZXL6AQAAQBAJ.

16. Gelman A, Fagan J, Kiss A. An analysis of the New York City police department's "stop-andfrisk" policy in the context of claims of racial bias. J Am Stat Assoc. 2007;102(479):813-823. doi:10.1198/016214506000001040

17. Chu H, Halloran ME. Bayesian estimation of vaccine efficacy. Clin Trials. 2004;1(3):306-314. doi:10.1191/1740774504cn025oa

18. Salvatier J, Wiecki T V., Fonnesbeck C. Probabilistic programming in Python using PyMC3. PeerJ Comput Sci. 2016;2:e55. doi:10.7717/peerj-cs.55

19. Parikh SR, Campbell H, Gray SJ, et al. Epidemiology, clinical presentation, risk factors, 
intensive care admission and outcomes of invasive meningococcal disease in England, 20102015. Vaccine. 2018;36(26):3876-3881. doi:10.1016/j.vaccine.2018.02.038

20. Argante L, Tizzoni M, Medini D. Fast and accurate dynamic estimation of field effectiveness of meningococcal vaccines. BMC Med. 2016;14(1):1-14. doi:10.1186/s12916-016-0642-2

21. Christensen H, Trotter CL, Hickman M, Edmunds WJ. Re-evaluating cost effectiveness of universal meningitis vaccination (Bexsero) in England: modelling study. Bmj. 2014;349(oct09 4):g5725-g5725. doi:10.1136/bmj.g5725

22. Beck E, Klint J, Garcia S, et al. Modelling the impact of 4CMenB and MenACWY meningococcal combined vaccination strategies including potential 4CMenB cross-protection: An application to England. Vaccine. 2020;38(47):7558-7568.

doi:10.1016/j.vaccine.2020.08.007

23. Vogel U, Taha M-K, Vazquez JA, et al. Predicted strain coverage of a meningococcal multicomponent vaccine (4CMenB) in Europe: a qualitative and quantitative assessment. Lancet Infect Dis. 2013;13(5):416-425. doi:10.1016/S1473-3099(13)70006-9

24. Parikh SR, Newbold L, Slater S, et al. Meningococcal serogroup B strain coverage of the multicomponent $4 \mathrm{CMenB}$ vaccine with corresponding regional distribution and clinical characteristics in England, Wales, and Northern Ireland, 2007-08 and 2014-15: a qualitative and quantitative assessment. Lancet Infect Dis. 2017;17(7):754-762. doi:10.1016/S14733099(17)30170-6

25. Medini D, Stella M, Wassil J. MATS: Global coverage estimates for 4 CMenB, a novel multicomponent meningococcal B vaccine. Vaccine. 2015;33(23):2629-2636. doi:10.1016/j.vaccine.2015.04.015

26. Rodrigues FMP, Marlow R, Simões MJ, Danon L, Ladhani S, Finn A. Association of Use of a Meningococcus Group B Vaccine With Group B Invasive Meningococcal Disease Among Children in Portugal. JAMA. 2020;324(21):2187. doi:10.1001/jama.2020.20449

27. Azzari C, Moriondo M, Nieddu F, et al. Effectiveness and impact of the 4cmenb vaccine against group b meningococcal disease in two italian regions using different vaccination 
schedules: A five-year retrospective observational study (2014-2018). Vaccines. 2020;8(3):112. doi:10.3390/vaccines 8030469

28. Marshall HS, McMillan M, Koehler AP, et al. Meningococcal B vaccine and meningococcal carriage in adolescents in Australia. N Engl J Med. 2020;382(4):318-327. doi:10.1056/NEJMoa1900236

29. Abad R, Medina V, Fariñas M del C, et al. Potential impact of the 4 CMenB vaccine on oropharyngeal carriage of Neisseria meningitidis. J Infect. 2017;75(6):511-520. doi:10.1016/j.jinf.2017.09.021

30. Domenech de Cellès M, Campbell H, Borrow R, Taha M-K, Opatowski L. Transmissibility and pathogenicity of the emerging meningococcal serogroup W sequence type-11 complex South American strain: a mathematical modeling study. BMC Med. 2020;18(1):1-12. doi:10.1186/s12916-020-01552-7 

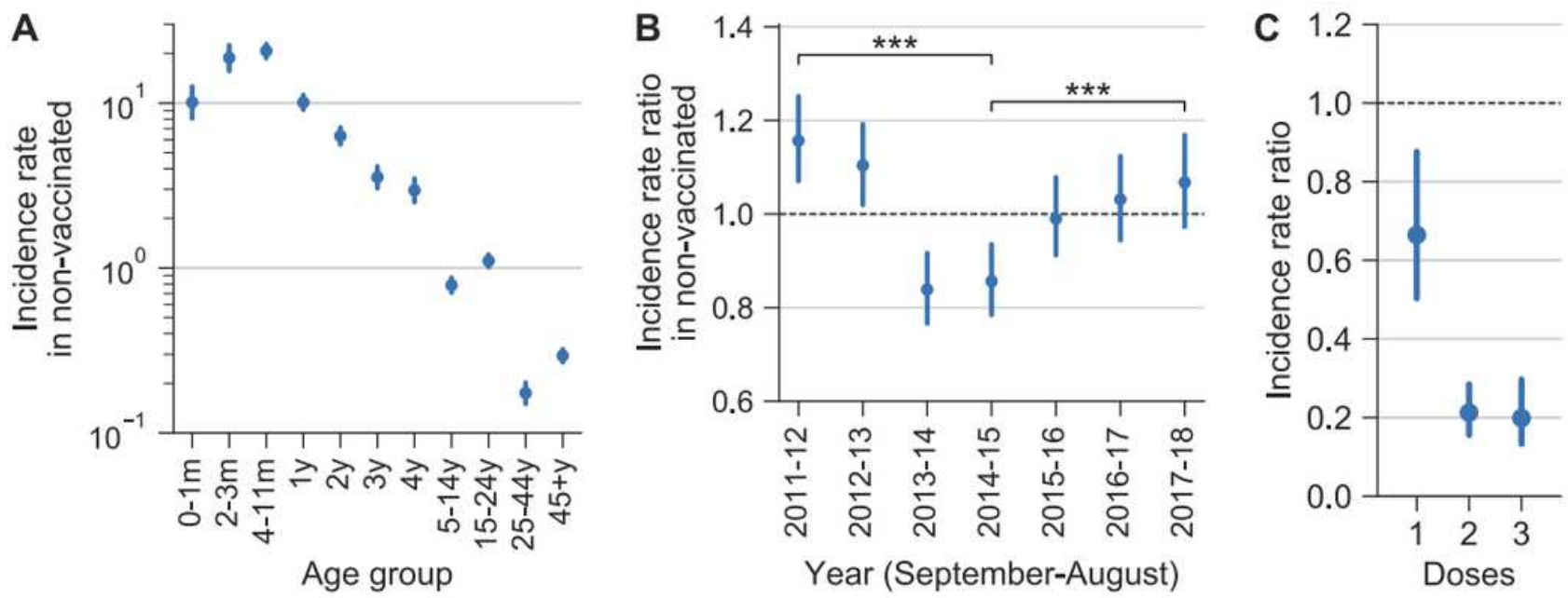

\section{Figure 1}

Please see the Manuscript PDF file for the complete figure caption
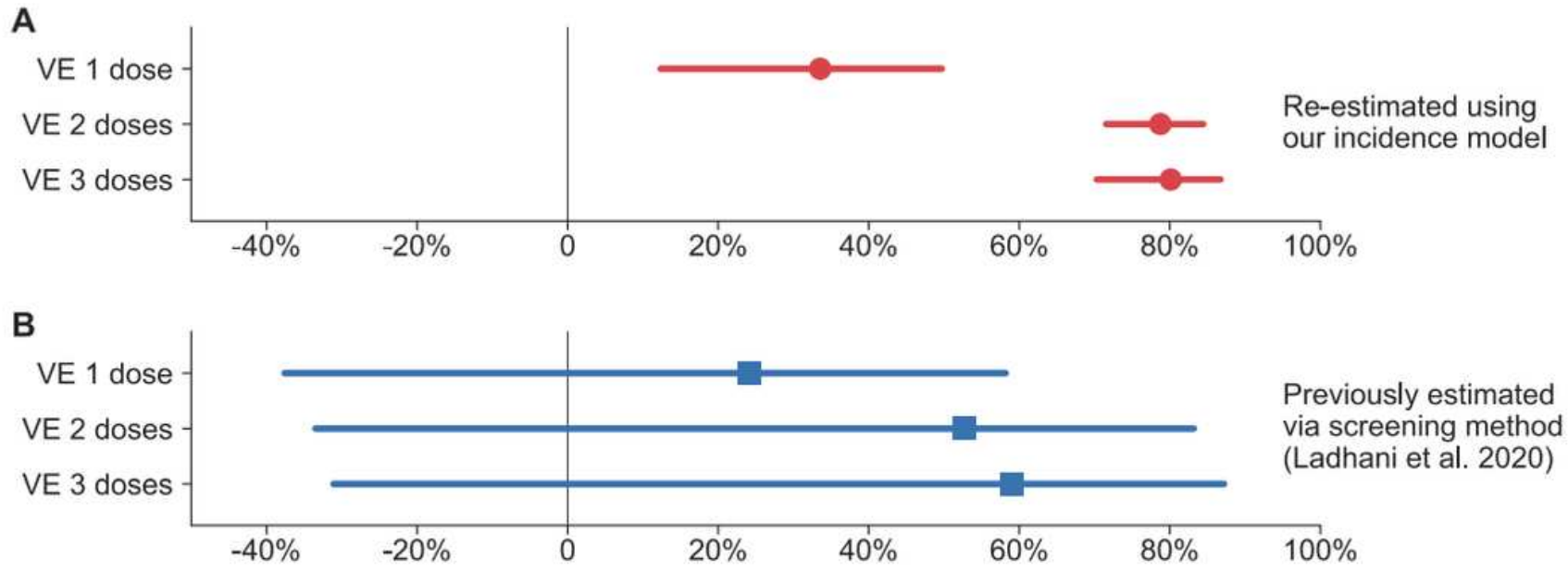

Figure 2

Please see the Manuscript PDF file for the complete figure caption 

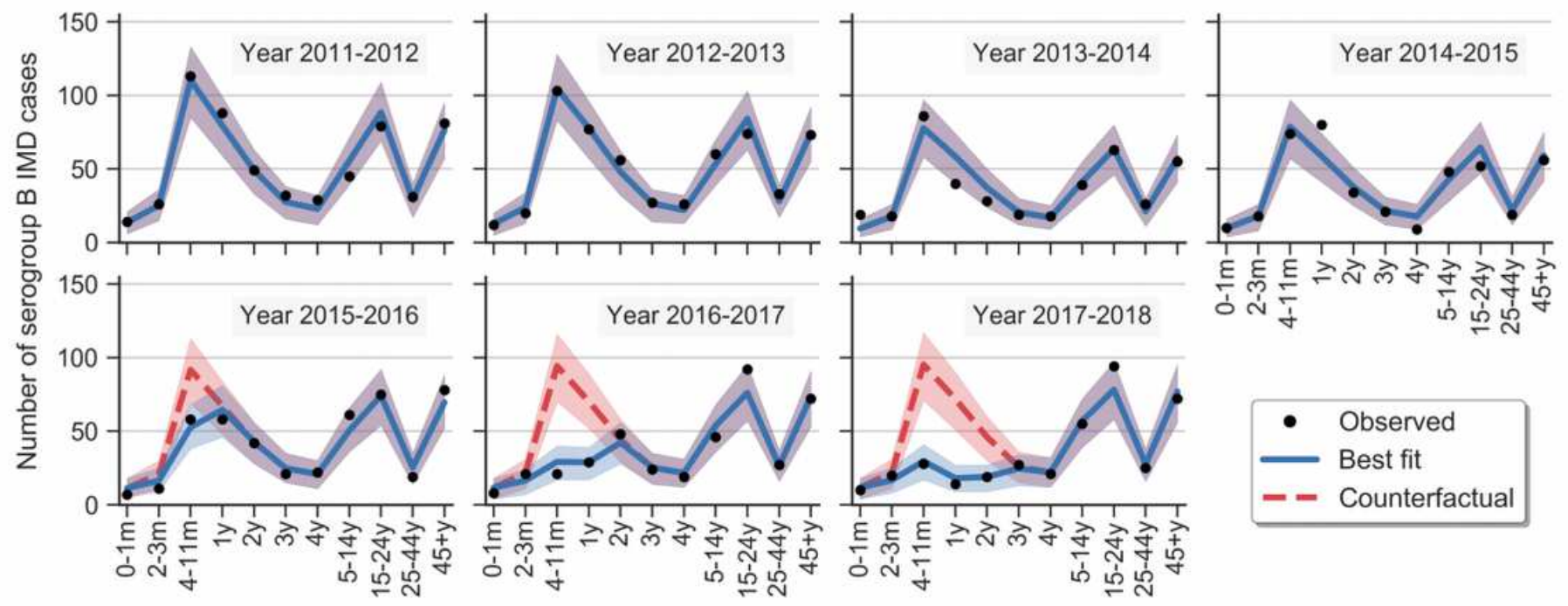

- Observed

- Best fit

- Counterfactual

Figure 3

Please see the Manuscript PDF file for the complete figure caption

\section{Supplementary Files}

This is a list of supplementary files associated with this preprint. Click to download.

- Argante4CMenBEffectivenessReestimationSupplementary.pdf 